\title{
In-situ Imaging of Electro-Chemo-Mechanical Degradation of High-Ni Content Cathode Materials
}

Huolin Xin ${ }^{1}$ and Chunyang Wang ${ }^{2}$

${ }^{1}$ University of California, Irvine, United States, ${ }^{2}$ University of California, Irvine, California, United States

High-Ni content and low-Co and No-Co cathodes have become the focus of research in the lithium-ion battery community, and have resurrected the interest in high-capacity layered lithium nickel oxide $\left(\mathrm{LiNiO}_{2}\right.$ aka LNO). Despite increased capacity and reduced cost, LNO-based cathodes suffer from rapid capacity fading and significant chemo-mechanical failures. Specifically, as lithium is extracted from LNO, the material experiences severe oxygen release and phase transformations, and develops cracks down to the atomic scales, leading to capacity fading and interface instabilities. In this talk, I will report our recent findings on the connection between oxygen release and LNO's mechanical degradation and a new method that inhibits both effects.

We show that $\mathrm{O} 1$ lattice blocks are formed in the delthiated LNO at high voltages. Under the hightemperature conditions, these $\mathrm{O} 1$ lattices transform into the rock salt structure through a complex atomicscale rearrangement which was captured by our atomic-scale observations. In addition, due to the oxygen loss, cracks are formed in LNO primary particles. By using electron tomography, we find two types of cracks, i.e., open and internal cracks, that have preferential alignment with the underlying crystal lattice. Due to the accelerated oxygen loss by the cracks, simultaneous rock-salt transformation was identified inside or around the cracks. In contrast to $\mathrm{LNO}$, we show that the doped $\mathrm{LiNiO}_{2}$ cathode shows reduced O1 lattice formation at high voltages, and the structural degradation toward the rock salt phase was significantly suppressed. In addition, because of the dopant effects, the number of internal cracks is largely reduced, and the propagation of surface-initiated cracks are suppressed. Our work provides a detailed understanding of the degradation mechanism of LNO and a large class of high-Ni content materials, and it also emphasizes the importance of mitigating O1 lattice, oxygen release, and cracks in high-Ni content cathodes. [1]

References

[1] This material is based upon work supported by the U.S. Department of Energy's Office of Energy Efficiency and Renewable Energy (EERE) under the Award Number: DE-EE0008444. 